\title{
Microfabricated Instrumentation for Chemical Sensing in \\ Industrial Process Control
}

\author{
J. Michael Ramsey \\ Oak Ridge National Laboratory \\ Oak Ridge, TN 37831-6142 \\ 865-574-5662
}

\section{CRADA FINAL REPORT}

Project Number: ORNL97-473 


\begin{abstract}
The monitoring of chemical constituents in manufacturing processes is of economic importance to most industries. The monitoring and control of chemical constituents may be of importance for product quality control or, in the case of process effluents, of environmental concern. The most common approach now employed for chemical process control is to collect samples which are returned to a conventional chemical analysis laboratory. This project will attempt to demonstrate the use of microfabricated structures, referred to as "lab-on-a-chip" devices, that accomplish chemical measurement tasks that emulate those performed in the conventional laboratory. The devices envisioned could be used as hand portable chemical analysis instruments where samples are analyzed in the field or as emplaced sensors for continuous "real-time" monitoring. This project will focus on the development of filtration elements and solid phase extraction elements that can be monolithically integrated onto electrophoresis and chromatographic structures pioneered in our laboratory. Successful demonstration of these additional functional elements on integrated microfabricated devices will allow labon-a-chip technologies to address real world samples that would be encountered in process control environments. The resultant technology will have broad application to industrial environmental monitoring problems such as monitoring municipal water supplies, waste water effluent from industrial facilities, or monitoring of run-off from agricultural activities. The technology will also be adaptable to manufacturing process control scenarios. Microfabricated devices integrating sample filtration, solid phase extraction, and chromatographic separation with solvent programming were demonstrated. Filtering of the sample was accomplished at the sample inlet with an array of seven channels each $1 \mu \mathrm{m}$ deep and $18 \mu \mathrm{m}$ wide. Sample concentration and separation were performed on channels $5 \mu \mathrm{m}$ deep and $25 \mu \mathrm{m}$ wide coated with a C18 phase, and elution was achieved under isocratic, step, or linear gradient conditions. For the solid phase extraction signal enhancement factors of 400 over a standard injection of $1.0 \mathrm{~s}$ were observed for a $320 \mathrm{~s}$ injection. Four polycyclic aromatic compounds (PAHs) were resolved by open channel electrochromatography in under $50 \mathrm{~s}$. Chip operation was unaffected by the presence of the $5 \mu \mathrm{m}$ silica particles at the filter entrance.
\end{abstract}




\section{Objectives}

Design, test, integrate and demonstrate significant functional elements to be included in microfabricated devices for process monitoring of aqueous streams. The significant objectives of this project include;

- a microfabricated sampling inlet that includes particle filtration to prevent fouling and solid phase extraction for sample preconcentration

- development of stationary phases for efficient electrokinetically driven chromatography and solid phase extraction.

- monolithically integrate the sampling inlet, and chemistries with microfabricated separations devices

- develop appropriate chemistries for at least one specific or group of target analytes and test on integrated device.

\section{Technical Achievements}

Advances in the fields of microfluidics and microinstrumentation in the past decade have given rise to microchips with broad ranges of functionality and versatility in the areas of chemical analysis. ${ }^{1-3}$ Primarily these areas have included separation methods such as electrophoresis, ${ }^{4-7}$ electrochromatography, ${ }^{8,9}$ micellar electrokinetic chromatography, ${ }^{10}$ isotachophoresis, ${ }^{11}$ isoelectric focusing, ${ }^{12}$ and capillary gel electrophoresis. ${ }^{13-16}$ In addition, the versatile nature of these devices with regards to design possibilities and flow control by electrokinetic means provides opportunities to perform multiple functions on one chip. In particular sample pretreatment procedures such as concentration enhancement and filtering can be important elements integrated on "lab-on-a-chip" devices.

Sample concentration is necessary in instances where trace analysis is desired, and the detection sensitivity is too low to reliably detect and quantify the analytes. Solid phase extraction is routinely used in liquid chromatographic methods for trace analysis in aqueous samples ${ }^{17-20}$ and has been used for the determination of polycyclic aromatic hydrocarbons in aqueous samples. ${ }^{21-26}$ On microchips, solid phase extraction has been demonstrated by coating the channel walls with a C18 phase ${ }^{27}$ and packing a channel with $\mathrm{C} 18$ coated $1.5-4 \mu \mathrm{m}$ particles. ${ }^{28}$ Other concentration techniques on microdevices include polymerase chain reaction $(\mathrm{PCR})^{29-34}$ and electrokinetic concentration at a porous membrane ${ }^{35}$ for the enhancement of DNA samples. Electric fields and various buffer systems have been used to effect sample stacking ${ }^{36,37}$ and isotachophoresis ${ }^{11}$ on microchips. For filtration, parallel slits have been used to capture red blood cells, ${ }^{38}$ a two dimensional array of posts to trap particulates, ${ }^{39}$ and diffusion-based transport to separate particles and molecules. $^{40,41}$

In this study, on-chip sample filtering, solid phase extraction, and open channel electrochromatography (OCEC) with solvent programming were demonstrated. The 

methanol. The chips were rinsed with methanol and stored in a methanol bath after each set of experiments.

Microchip operation. Prior to analysis the chips were rinsed with a $10 \mathrm{mM}$ TRIS buffer with $20 \%$ acetonitrile $(\mathrm{v} / \mathrm{v})$. The sample was placed in the sample reservoir with or without $5 \mu \mathrm{m}$ silica particles (YMC, Inc, Morris Plains, NJ). $10 \mathrm{mM}$ TRIS buffers with $20 \%$ acetonitrile and $60 \%$ acetonitrile were placed in the buffer 1 and 2 reservoirs, respectively. $10 \mathrm{mM}$ TRIS buffer with $20 \%$ acetonitrile was placed in the two remaining reservoirs, and all reservoirs were capped with white rubber septa (Aldrich) to minimize evaporation. Five independent high voltage power supplies (10A12-P4; Ultravolt, Ronkonkoma, NY) provided the potential for the reservoirs. Each source was computer controlled using multifunction I/O cards (PCI-M1016-XE50; National Instruments, Austin, TX) in a Power Macintosh 7500/100 running LabView (National Instruments). Platinum wire electrodes were inserted through the septa to make electrical contact with the buffer solutions. All buffers were prepared from a stock TRIS buffer solution made from 99.9\% TRIZMA base (Sigma, St. Louis, MO) dissolved in Nanopure purified water (Barnstead, Dubuque, IA) and adjusted to $\mathrm{pH} 8.2$ with $\mathrm{HCl}$ (Fisher Scientific, Fairlawn, NJ).

The computer controlled injection and elution programs were set up to (a) hold the equilibration conditions, (b) inject the sample for a preset time, and (c) begin the elution scheme for the remaining analysis time. The applied potentials are listed in Table 2. Buffers 1 and 2 were mixed electroosmotically at the T-intersection ${ }^{9,10}$ by keeping the potential at the T-intersection constant and adjusting the potentials applied to the buffer 1 and 2 reservoirs. For these experiments, the mixing range of buffer 2 with buffer 1 was $10 \%-90 \%$ and allowed a minimum and maximum acetonitrile concentration for elution of $24 \%$ and $56 \%$, respectively. This range of organic concentration provided the necessary selectivity for the samples tested. Prior to injection the analysis channel was equilibrated with $10 \mathrm{mM}$ TRIS buffer with $24 \%(\mathrm{v} / \mathrm{v})$ acetonitrile. With the gated injection method, ${ }^{43}$ samples were injected onto the analysis channel by lowering the voltages at the buffer 1 and 2 reservoirs for the desired injection time. This allowed the sample to be transported electroosmotically onto the analysis channel. At the end of the injection time, flow from the buffer 1 and 2 channels was reestablished terminating sample flow onto the analysis channel. During the injection, the voltage settings produced a field strength of $350 \mathrm{~V} / \mathrm{cm}$ in the analysis channel. The isocratic elution used $56 \%$ acetonitrile over the course of the separation. The step gradient was held at $52 \%$ acetonitrile for the first $10 \mathrm{~s}$ of the elution scheme and then switched to $56 \%$ acetonitrile for the remainder of the analysis time. For the linear gradient the elution program started at $24 \%$ acetonitrile and ramped in $1 \%$ increments to $56 \%$ acetonitrile over $16 \mathrm{~s}$. A field strength of $500 \mathrm{~V} / \mathrm{cm}$ along the analysis channel was maintained for all of the elution processes. Due to slight variations in channel geometries the applied potentials to chip 2 differed nominally from those applied to chip 1. Note that for all settings for injection and elution, the sample reservoir maintained the highest applied voltage setting. This design would allow the chip to be operated with an alternative voltage scheme with the sample reservoir at ground and the remaining reservoirs set to a negative potential. Samples of various concentrations in 10 mM TRIS with $20 \%(\mathrm{v} / \mathrm{v})$ acetonitrile were prepared from stock solutions of anthracene $98 \%$, pyrene $99+\%, 1,2$ benzofluorene $99+\%$, (Aldrich, Milwaukee, WI) and benzo(a)pyrene (Eastman Kodak, Rochester, NY) in acetonitrile. Many PAHs are 
carcinogenic, and care must be taken when handling these compounds. Consult the appropriate Material Safety Data Sheets for additional information.

Detection. Laser-induced fluorescence (LIF) was used for sample detection. The $325 \mathrm{~nm}$ line of a He-Cd laser (2074-M-A02; Melles Griot, Carlsbad, CA) was focused using a $200 \mathrm{~mm}$ focal length lens on the analysis channel at a point $29 \mathrm{~mm}$ from the injection valve. Fluorescence emission, collected using a 40x microscope objective, was focused on an $800 \mu \mathrm{m}$ pinhole, spectrally filtered using a $350 \mathrm{~nm}$ longpass filter, and then measured by a photomultiplier tube (PMT, 77348 Oriel Instruments, Inc., Stratford, CT). The PMT signal was fed through a current amplifier (428; Keithley Instruments, Inc., Cleveland, $\mathrm{OH}$ ) with a $10 \mathrm{~ms}$ filter. Data were collected at $25 \mathrm{~Hz}$ or $50 \mathrm{~Hz}$ by the same multifunction I/O card used for voltage control, and the LabView program used for voltage control also served for data acquisition.

Images of regions of interest on the chip were taken using a CCD camera (TE/CCD-512TKM; Princeton Instruments, Inc., Trenton, NJ) on an inverted microscope (TE300; Nikon, Inc., Melville, NY) at 20x magnification. IPLab Software (Signal Analytics Corp., Vienna, VA) was used for image acquisition and camera operation. A neutral marker rhodamine B (Eastman Kodak) in $10 \mathrm{mM}$ TRIS buffer with $20 \%(\mathrm{v} / \mathrm{v})$ acetonitrile was used for imaging fluid flow to optimize sample injections at the valve and to determine mixing ratios at the T-intersection for a range of applied voltages.

\section{Results and Discussion}

Pyrene at $0.028,0.28$, and $2.8 \mu \mathrm{M}$ was concentrated with increasing injection times and subsequently eluted under isocratic conditions at $56 \%$ acetonitrile on chip 1. The chromatograms for the $2.8 \mu \mathrm{M}$ concentration, ranging from 20 to $560 \mathrm{~s}$ injection times, were overlaid and are shown in Figure $2 a$. Figures $2 b$ and $2 c$ show the peak heights and peak areas, respectively, for each concentration normalized to their largest value in the injection time range. All three concentrations showed a maximum in peak height in the 300 to $480 \mathrm{sec}$ injection time range. The sublinear growth, and eventual decrease, of peak heights with increasing injection time may be due to a reduction in the sharpness of the eluting solvent front. The fluid flow from the buffer 1 and 2 and $\mathrm{C}$ channels (Figure 1) was minimized during injection resulting in diffusive mixing of the two buffers in the $\mathrm{C}$ channel. Consequently, when the microchip was switched from the inject to elute mode, the leading edge of the eluting buffer became more diffuse with longer injection times. This was seen in the fronting of the eluted peaks for the injection times $>400 \mathrm{~s}$ and was not pronounced for injection times $<360 \mathrm{~s}$. Despite this trend the peak areas (normalized to their maximum peak area) showed a linear increase with injection time leading to a plateau (Figure 2c). There was a finite amount of stationary phase in the analysis channel, and the amount of material that could be concentrated reached a maximum after which no additional sample could be accumulated on the stationary phase. The time to reach this plateau for a given compound increased with decreasing concentration as seen in Figure 2c. This system is similar to open tubular systems, and consequently, the stationary phase density is lower than with packed beds. If necessary, future systems could potentially incorporate monolithic stationary phases to increase the stationary phase density.

To determine the signal enhancement factor, pyrene $(900 \mathrm{nM})$ was concentrated with increasing injection times up to $320 \mathrm{~s}$ and compared to a standard $1 \mathrm{~s}$ injection. For 
the $1 \mathrm{~s}$ injections the analysis channel was equilibrated with $56 \%$ acetonitrile instead of $24 \%$ and eluted with $56 \%$ acetonitrile. The chromatograms for a $1 \mathrm{~s}$ injection and a $320 \mathrm{~s}$ concentration time are shown in Figure 3 . The $1 \mathrm{~s}$ injection barely registers as a disturbance on the same scale as the $320 \mathrm{~s}$ injection, and therefore, the chromatogram was rescaled and included as an inset (a). For each injection time an enhancement factor was calculated by dividing the baseline corrected peak height by the average height for the $1 \mathrm{~s}$ injections. The enhancement factor with injection time was linear with a correlation coefficient of 0.995 , and the enhancement factor for the $320 \mathrm{~s}$ injection data was 400 . (The data were smoothed using a $2^{\text {nd }}$ order, 11 point, Savitzky-Golay function.)

To demonstrate the feasibility of separating a concentrated sample on the channel, $20 \mathrm{~s}$ injections of a four component mixture were made in two concentration ranges on chip 1. These injections were made without silica particles present at the filter. The resulting chromatograms are shown in Figure 4. The sample was comprised of anthracene $(2.8 \mu \mathrm{M})$, pyrene $(0.9 \mu \mathrm{M}), 1,2$ benzofluorene $(5.8 \mu \mathrm{M})$, and benzo(a)pyrene $(5.0 \mu \mathrm{M})$ in Figure $4 \mathrm{a}$, and anthracene $(100 \mathrm{nM})$, pyrene $(28 \mathrm{nM}), 1,2$ benzofluorene (230 $\mathrm{nM})$, and benzo(a)pyrene $(200 \mathrm{nM})$ in Figure $4 \mathrm{~b}$. The step gradient used after injection was $52 \%$ acetonitrile for $10 \mathrm{~s}$ and then switched to $56 \%$ acetonitrile for the remainder of the analysis to achieve baseline resolution. The data in Figures $4 \mathrm{a}$ and $\mathrm{b}$ were obtained on different microchips, and the variability in fabrication and channel coating from chip to chip resulted in a difference in retention times. These variations can be normalized through appropriate calibration. Limits of detection (LOD, set at $3 \sigma$ ) were calculated from smoothed data for the compounds in Figure $4 \mathrm{~b}$ and were $3.1 \mathrm{nM}$ for anthracene, 1.0 $\mathrm{nM}$ for pyrene, $8.1 \mathrm{nM}$ for 1,2 benzofluorene, and $17 \mathrm{nM}$ for benzo(a)pyrene for a $20 \mathrm{~s}$ concentration time. The LOD of pyrene for this $20 \mathrm{~s}$ concentration time is 48 times lower than the LOD of the $1 \mathrm{~s}$ standard injection without concentration ( $48 \mathrm{nM}$, Figure $3 \mathrm{a})$. This value matches well with the enhancement factor of 49 calculated for the $20 \mathrm{~s}$ concentration time and is included in the series shown in Figure 2a. Extrapolating using an enhancement factor of 400 for the 320 s concentration time, an LOD of $100 \mathrm{pM}$ for pyrene should be attainable.

Chip operation was then tested with $5 \mu \mathrm{m}$ silica particles added to the sample reservoir to act as a particulate contaminant. These particles were effectively blocked from entering the sample channels as shown in Figure 5. At 20x magnification the seven channels comprising the filter element could not be completely imaged and, therefore, three frames were taken to form the composite image. To test the effect of these particles on chip operation, a sample of rhodamine B prepared in buffer with $20 \%$ acetonitrile was used to image the valving operation at the cross. Valve operation was observed to be unaffected by the presence of the particles.

To improve control of the mixing of buffers 1 and 2 at the T-intersection in chip 2 (Figure 1), the narrow sections of the buffer 1 and 2 channels were lengthened. This allowed finer control of the electric field and, hence, finer control of the mixing of the two streams. The separation of four PAHs with $5 \mu \mathrm{m}$ silica present as the solid contaminant was repeated on chip 2 using three elution schemes to demonstrate the effect of the particles on peak resolution. The components and their concentrations in the sample were $2.8 \mu \mathrm{M}$ anthracene, $0.90 \mu \mathrm{M}$ pyrene, $5.8 \mu \mathrm{M} 1,2$ benzofluorene, and $5.0 \mu \mathrm{M}$ benzo(a)pyrene in buffer with $20 \%$ acetonitrile. The chromatograms are shown in Figure 6. An isocratic elution at $52 \%$ acetonitrile provided good resolution of all four 
compounds (Figure 6a). A step gradient beginning at 52\% acetonitrile, holding for $10 \mathrm{~s}$, and ramping to $56 \%$ acetonitrile gave similar resolution (Figure 6b). However, the step gradient sharpened the peak for the last eluting compound, benzo(a)pyrene, compared to the isocratic elution in Figure 6a. The linear gradient shown in Figure 6c improved separation between the first and second compound, worsened the peak shape of the last eluting compound, increased the overall analysis time by $\sim 10 \mathrm{~s}$, but provided an increased peak capacity (see below). The linear gradient was not optimized for the sample studied here. Efficiency values, $\mathrm{N}$, for the peaks in Figure 6a, were calculated from their $2^{\text {nd }}$ moment. These values and the plate heights, $\mathrm{H}$, are shown in Table 3 . In addition, peak capacities were determined for Figure 6a, b, and c and were 26, 30, and 45, respectively, for a resolution, $\mathrm{R}$, of 1.0 . The peak capacity for Figure $6 \mathrm{~b}$ (with particles at the filter) compares favorably to the peak capacities of 37 and 31 (without particles) calculated for Figure $4 \mathrm{a}$ and b, respectively. Isocratic and step gradient elution were, in this case, more than capable of providing acceptable results and in less time. Sample loading capacities were determined from breakthrough volume data generated by injecting each sample for an extended period of time (e.g., $>200 \mathrm{~s})$ until equilibration of the sample with the entire channel length was achieved. Loading capacities were calculated from the resulting breakthrough curves and were $78 \mathrm{fmol}$ (anthracene), 110 fmol (pyrene), $130 \mathrm{fmol}$ (1,2 benzofluorene), and $180 \mathrm{fmol}$ (benzo(a)pyrene).

Using the step gradient elution method, the effect of concentration time on the separation was considered. Figure 7a, 7b, and 7c show the chromatograms for 80, 160, and $320 \mathrm{~s}$ injections, respectively, while filtering $5 \mu \mathrm{m}$ silica at the sample reservoir as shown in Figure 5. With increasing concentration time an increase in both peak height and width was observed for the first sample peak, anthracene. The peak shapes of anthracene and pyrene show some degree of deformation as the injection time approached and/or surpassed their respective breakthrough times $(210 \mathrm{~s}$ for anthracene and $400 \mathrm{~s}$ for pyrene). The peak variance for increasing concentration time is presented in Figure 8. The peak variances were calculated assuming a Gaussian distribution for all sample peaks except for the first eluting peak (anthracene) concentrated for 160 and $320 \mathrm{~s}$ where a rectangular distribution was assumed. ${ }^{44}$ Where necessary, the peak width was extrapolated to baseline. As expected the peak variances hold steady and then begin to increase as more sample was concentrated with increasing injection time. Maximum concentration without additional dispersion for all four compounds was located at the 80 s concentration time.

In conclusion, filtration, concentration, and separation of a four component mixture were successfully performed on a quartz microchip. Filtration of solid particles was achieved without a detrimental impact on chip operation and performance. Reasonable efficiencies for isocratic elution runs were obtained despite having a relatively short separation channel $(29 \mathrm{~mm})$ and limited stationary phase density. Further studies on monolithic phases generated in the channel will be conducted to improve concentration factors, increase stationary phase density, and examine separation performance.

\section{Inventions Reported:}


Invention Disclosure ID0975 entitled, "Sample Filtration, Concentration, and Separation on Microchips," was filed by Stephen C. Jacobson and J. Michael Ramsey

\section{Commercial Possibilities:}

Devices similar to those developed in this program have commercial application in the monitoring of organic species in aqueous media. Waters' interest resulted in there formation of a separate company, Protasis, for developing such applications. These devices, with some modification and further development, also have application in the pharmaceutical development process. Examples include performing drug metabolism and pharmaco-kinetic studies.

\section{Plans for Future Collaborations}

A follow-on CRADA, ORNL 01-0628, has been executed with Waters for the investigation of interfacing technologies such as developed in this program to mass spectrometry. The primary applications relate to the problems in the pharmaceutical industry. 
Table 1. Channel lengths.

\begin{tabular}{|c|c|c|c|c|c|c|c|c|c|c|}
\hline & & \multicolumn{8}{|c|}{ Channel } \\
\hline & & Chip & B1 & B2 & $\mathrm{S}$ & SW & $\mathrm{AW}$ & $\mathrm{C}$ & Filter & Filter* \\
\hline \multirow{3}{*}{$\begin{array}{c}\text { Length } \\
(\mathrm{mm})\end{array}$} & Narrow & 1 & 1.0 & 1.0 & 24.7 & 15.2 & 30 & 5.0 & 2.5 & 2.0 \\
\hline & & 2 & 8.3 & 8.0 & 24.7 & 15.2 & 30 & 5.0 & 2.5 & 2.4 \\
\hline & Wide & $\begin{array}{l}1 \\
2\end{array}$ & $\begin{array}{l}9.5 \\
-\end{array}$ & $\begin{array}{c}9.3 \\
-\end{array}$ & - & $\begin{array}{l}8.4 \\
8.7\end{array}$ & $\begin{array}{l}15 \\
13\end{array}$ & - & - & - \\
\hline
\end{tabular}

*Denotes the shallow portion of the filter element.

Table 2. Potentials applied to the buffer 1 and 2 reservoirs.

\begin{tabular}{|c|c|c|c|c|}
\cline { 2 - 5 } \multicolumn{1}{c|}{} & \multicolumn{2}{c|}{ Chip 1 } & \multicolumn{2}{c|}{ Chip 2 } \\
\hline Operating condition & $\begin{array}{c}\text { Buffer 1 } \\
(\mathbf{k V})\end{array}$ & $\begin{array}{c}\text { Buffer 2 } \\
(\mathbf{k V})\end{array}$ & $\begin{array}{c}\text { Buffer 1 } \\
(\mathbf{k V})\end{array}$ & $\begin{array}{c}\text { Buffer 2 } \\
(\mathbf{k V})\end{array}$ \\
\hline equilibrate & 2.04 & 1.96 & 2.10 & 1.90 \\
\hline inject & 1.14 & 1.14 & 1.12 & 1.12 \\
\hline $24 \% \mathrm{ACN}$ & 2.04 & 1.96 & 2.10 & 1.90 \\
\hline $52 \% \mathrm{ACN}$ & 1.97 & 2.03 & 1.84 & 2.16 \\
\hline $56 \% \mathrm{ACN}$ & 1.93 & 2.07 & 1.80 & 2.20 \\
\hline
\end{tabular}

Potentials at the sample, sample waste, and analysis waste reservoirs were held constant at 2.2, 1.0 (0.80 for chip 2), and $0.0 \mathrm{kV}$, respectively.

Table 3. Separation efficiencies for Figure 6a.

\begin{tabular}{|l|c|c|}
\hline \multicolumn{1}{|c|}{ Peak } & Plate Number, $\mathbf{N}$ & $\begin{array}{c}\text { Plate height, } \mathbf{H} \\
(\mu \mathbf{m})\end{array}$ \\
\hline 1- Anthracene & 31800 & 0.912 \\
\hline 2- Pyrene & 31600 & 0.918 \\
\hline 3- 1,2 Benzofluorene & 25100 & 1.16 \\
\hline 4- Benzo(a)pyrene & 3320 & 8.74 \\
\hline
\end{tabular}

\section{References}

(1) Colyer, C. L.; Tang, T.; Chiem, N.; Harrison, D. J. Electrophoresis 1997, 18, 17331741.

(2) Effenhauser, C. S.; Bruin, G. J. M.; Paulus, A. Electrophoresis 1997, 18, 22032213.

(3) Ramsey, J. M. Nature Biotech. 1999, 17, 1061-1062.

(4) Manz, A.; Harrison, D. J.; Verpoorte, E. M. J.; Fettinger, J. C.; Paulus, A.; Lüdi, H.; Widmer, H. M. J. Chromatogr. 1992, 593, 253-258. 
(5) Harrison, D. J.; Fluri, K.; Seiler, K.; Fan, Z.; Effenhauser, C. S.; Manz, A. Science 1993, 261, 895-897.

(6) Effenhauser, C. S.; Manz, A.; Widmer, H. M. Anal. Chem. 1993, 65, 2637-42.

(7) Jacobson, S. C.; Hergenröder, R.; Koutny, L. B.; Ramsey, J. M. Anal. Chem. 1994, 66, 1114-1118.

(8) He, B.; Tait, N.; Regnier, F. Anal. Chem. 1998, 70, 3790-3797.

(9) Kutter, J. P.; Jacobson, S. C.; Matsubara, N.; Ramsey, J. M. Anal. Chem. 1998, 70, 3291-3297.

(10) Kutter, J. P.; Jacobson, S. C.; Ramsey, J. M. Anal. Chem. 1997, 69, 5165-5171.

(11) Walker, P. A., III; Morris, M. D.; Burns, M. A.; Johnson, B. N. Anal. Chem. 1998, 70, 3766-3769.

(12) Hofmann, O.; Che, D.; Cruickshank, K. A.; Müller, U. R. Anal. Chem. 1999, 71, 678-686.

(13) Woolley, A. T.; Mathies, R. A. Proc. Natl. Acad. Sci. U. S. A. 1994, 91, $11348-$ 11352.

(14) Effenhauser, C. S.; Paulus, A.; Manz, A.; Widmer, H. M. Anal. Chem. 1994, 66, 2949-2953.

(15) Schmalzing, D.; Adourian, A.; Koutny, L.; Ziaugra, L.; Matsudaira, P.; Ehrlich, D. Anal. Chem. 1998, 70, 2303-2310.

(16) Waters, L. C.; Jacobson, S. C.; Kroutchinina, N.; Khandurina, J.; Foote, R. S.; Ramsey, J. M. Anal. Chem. 1998, 70, 5172-5176.

(17) Berrueta, L. A.; B.Gallo; Vicente, F. Chromatographia 1995, 40, 474-481.

(18) Font, G.; Mañes, J.; Moltó, J. C.; Picó, Y. J. Chromatogr. 1993, 642, 135-161.

(19) Hennion, M.-C. J. Chromatogr. A 1999, 856, 3-54.

(20) Subra, P.; Hennion, M.-C.; Rosset, R.; Frei, R. W. J. Chromatogr. A 1988, 456, 121-141.

(21) Bouzige, M.; Pichon, V.; Hennion, M.-C. J. Chromatogr. A 1998, 823, 197-210.

(22) Furton, K. G.; Jolly, E.; Pentzke, G. J. Chromatogr. 1993, 642, 33-45.

(23) Kicinski, H. G.; Adamek, S.; Kettrup, A. Chromatographia 1989, 28, 203-208.

(24) Kiss, G.; Varga-Puchony, Z.; Hlavay, J. J. Chromatogr. A 1996, 725, 261-272.

(25) Nirmaier, H.-P.; Fischer, E.; Meyer, A.; Henze, G. J. Chromatogr. A 1996, 730, 169-175.

(26) Vera-Avila, L. E.; Covarrubias, R. Intern. J. Environ. Anal. Chem. 1994, 56, 33-47.

(27) Kutter, J. P.; Jacobson, S. C.; Ramsey, J. M. J. Microcolumn Sep. 2000, 12, 93-97.

(28) Oleschuk, R. D.; Shultz-Lockyear, L. L.; Ning, Y.; Harrison, D. J. Anal. Chem. 2000, 72, 585-590.

(29) Ibrahim, M. S.; Lofts, R. S.; Jahrling, P. B.; Henchal, E. A.; Weedn, V. W.; Northrup, M. A.; Belgrader, P. Anal. Chem. 1998, 70, 2013-2017.

(30) Kopp, M. U.; Mello, A. J. d.; Manz, A. Science 1998, 280, 1046-1048.

(31) Northrup, M. A.; Benett, B.; Hadley, D.; Landre, P.; Lehew, S.; Richards, J.; Stratton, P. Anal. Chem. 1998, 70, 918-922.

(32) Ross, P. L.; Davis, P. A.; Belgrader, P. Anal. Chem. 1998, 70, 2067-2073.

(33) Waters, L. C.; Jacobson, S. C.; Kroutchinina, N.; Khandurina, J.; Foote, R. S.; Ramsey, J. M. Anal. Chem. 1998, 70, 158-162.

(34) Lagally, E. T.; Medintz, I.; Mathies, R. A. Anal. Chem. 2001, 73, 565-570. 
(35) Khandurina, J.; Jacobson, S. C.; Waters, L. C.; Foote, R. S.; Ramsey, J. M. Anal. Chem. 1999, 71, 1815-1819.

(36) Jacobson, S. C.; Ramsey, J. M. Electrophoresis 1995, 16, 481-486.

(37) Kutter, J. P.; Ramsey, R. S.; Jacobson, S. C.; Ramsey, J. M. J. Microcolumn Sep. 1998, 10, 313-319.

(38) Wilding, P.; Pfahler, J.; Bau, H. H.; Zemel, J. N.; Kricka, L. J. Clin. Chem. 1994, $40,43-47$.

(39) He, B.; Tan, L.; Regnier, F. Anal. Chem. 1999, 71, 1464-1468.

(40) Brody, J. P.; Yager, P. Sensors and Actuators A 1997, 58, 13-18.

(41) Weigl, B. H.; Yager, P. Science 1999, 283, 346-347.

(42) Khandurina, J.; Jacobson, S. C.; Waters, L. C.; Foote, R. S.; Ramsey, J. M. Anal. Chem. 1999, 71, 1815-1819.

(43) Jacobson, S. C.; Koutny, L. B.; Hergenröder, R.; Moore, A. W., Jr.; Ramsey, J. M. Anal. Chem. 1994, 66, 3472-3476.

(44) Landers, J. P. In Handbook of Capillary Electrophoresis; CRC Press: New York, 1997, pp 894. 
(a)

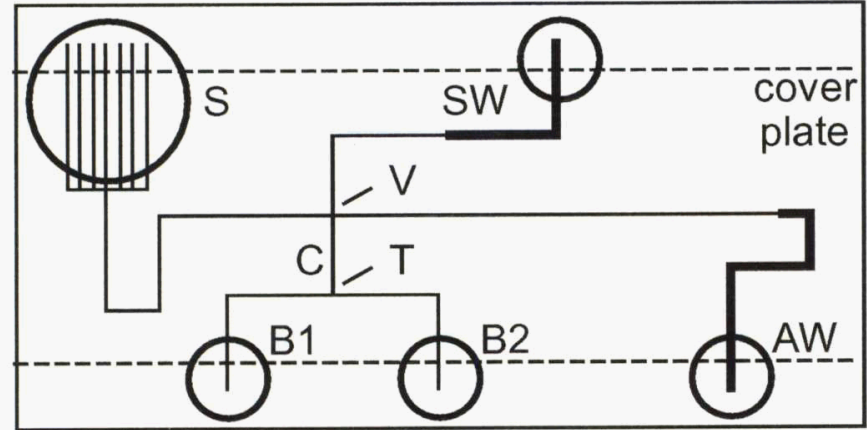

(b)

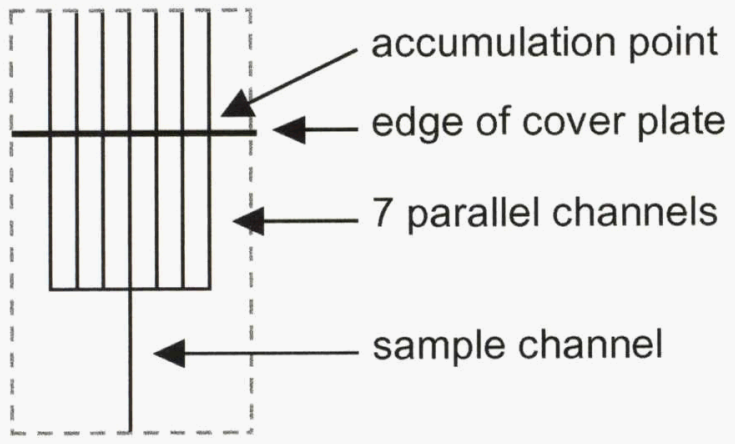

(c)

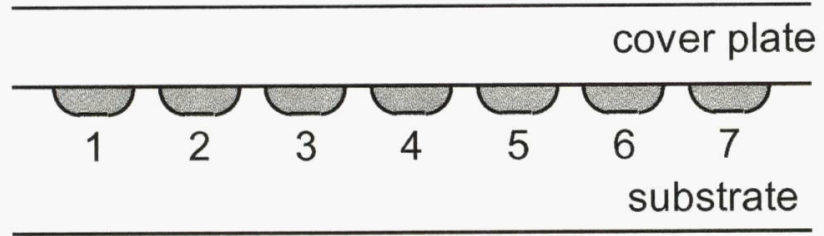

Figure 1. (a) A schematic of the microchip for sample filtration, concentration, and separation. The labels are (B1) buffer with $20 \%$ acetonitrile, (B2) buffer with $60 \%$ acetonitrile, (S) sample, (SW) sample waste, (AW) analysis waste, (T) T-intersection, and (V) valve. The B1 and B2 channels on Chip 1 have wide and narrow portions, and the B1 and $\mathrm{B} 2$ channels on Chip 2 are uniformly narrow. The circles represent fluid reservoirs. (b) An expanded view and (c) cross section of the filter element. The filter channels are numbered 1 to 7 . See text and Table 1 for channel dimensions. Schematics are not to scale. 


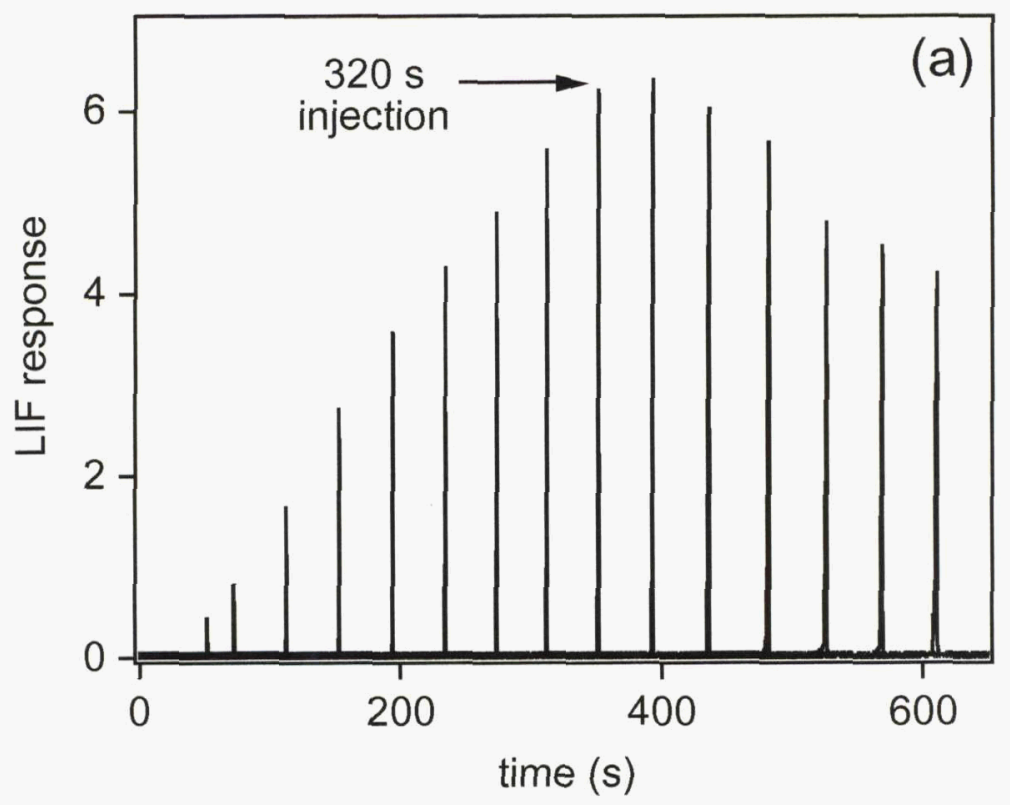

Figure 2a. Overlaid chromatograms for injection times of $20 \mathrm{~s}$ and 40 to $560 \mathrm{~s}$ in $40 \mathrm{~s}$ increments. The time on the $\mathrm{x}$-axis is the injection time plus the elution time. Chip 1 and isocratic elution were used, and the sample was $2.8 \mu \mathrm{M}$ pyrene. 


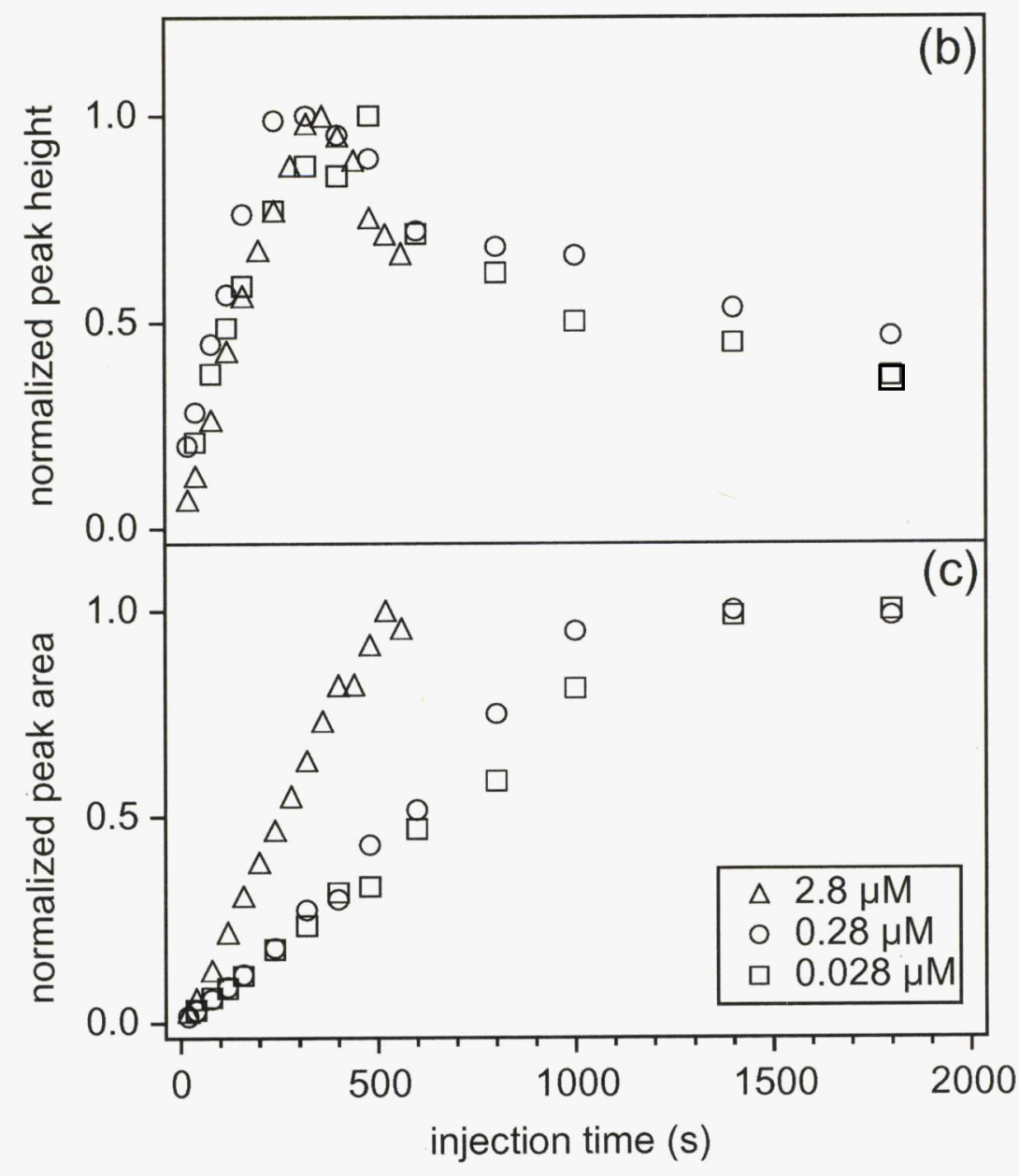

Figures $2 b$ and $2 c$. Variation of the (b) peak heights and (c) peak areas with injection time. The peak heights and areas were normalized to the largest value in each data set. 


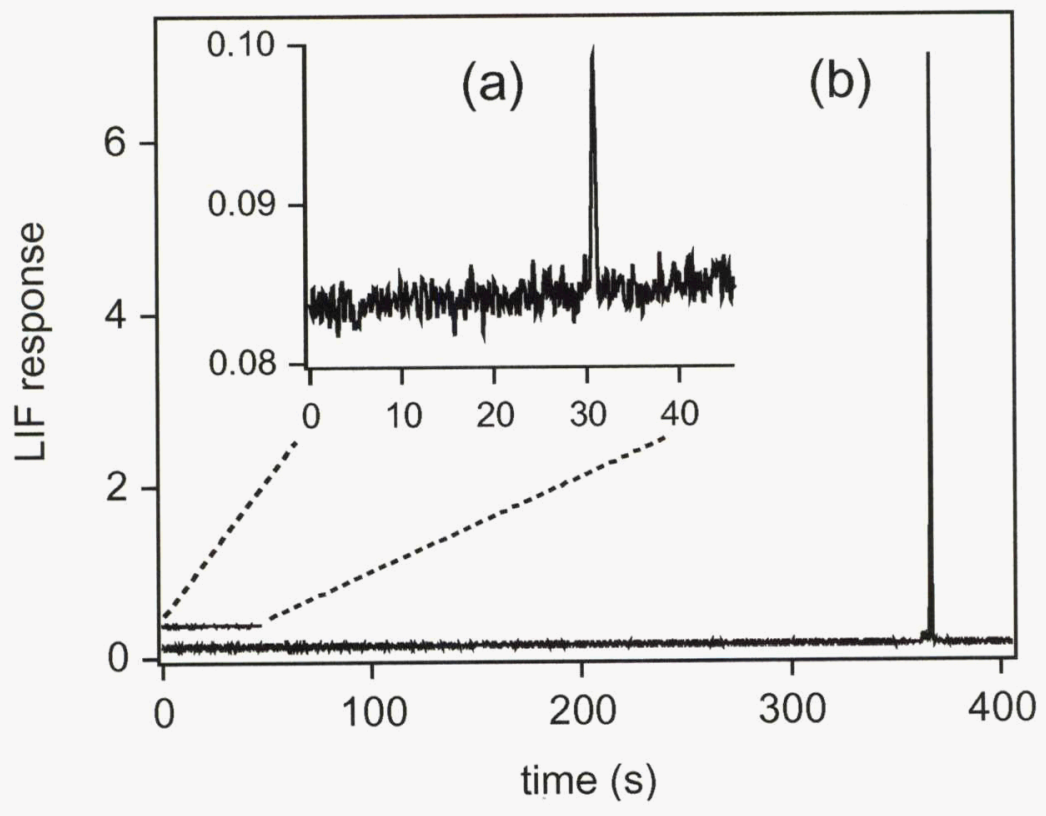

Figure 3. Chromatograms of (a) $1 \mathrm{~s}$ injection without concentration and (b) $320 \mathrm{~s}$ injection with concentration. (a) is offset from (b) and also inset for clarity. Chip 1 was used, and the sample was $900 \mathrm{nM}$ pyrene. 


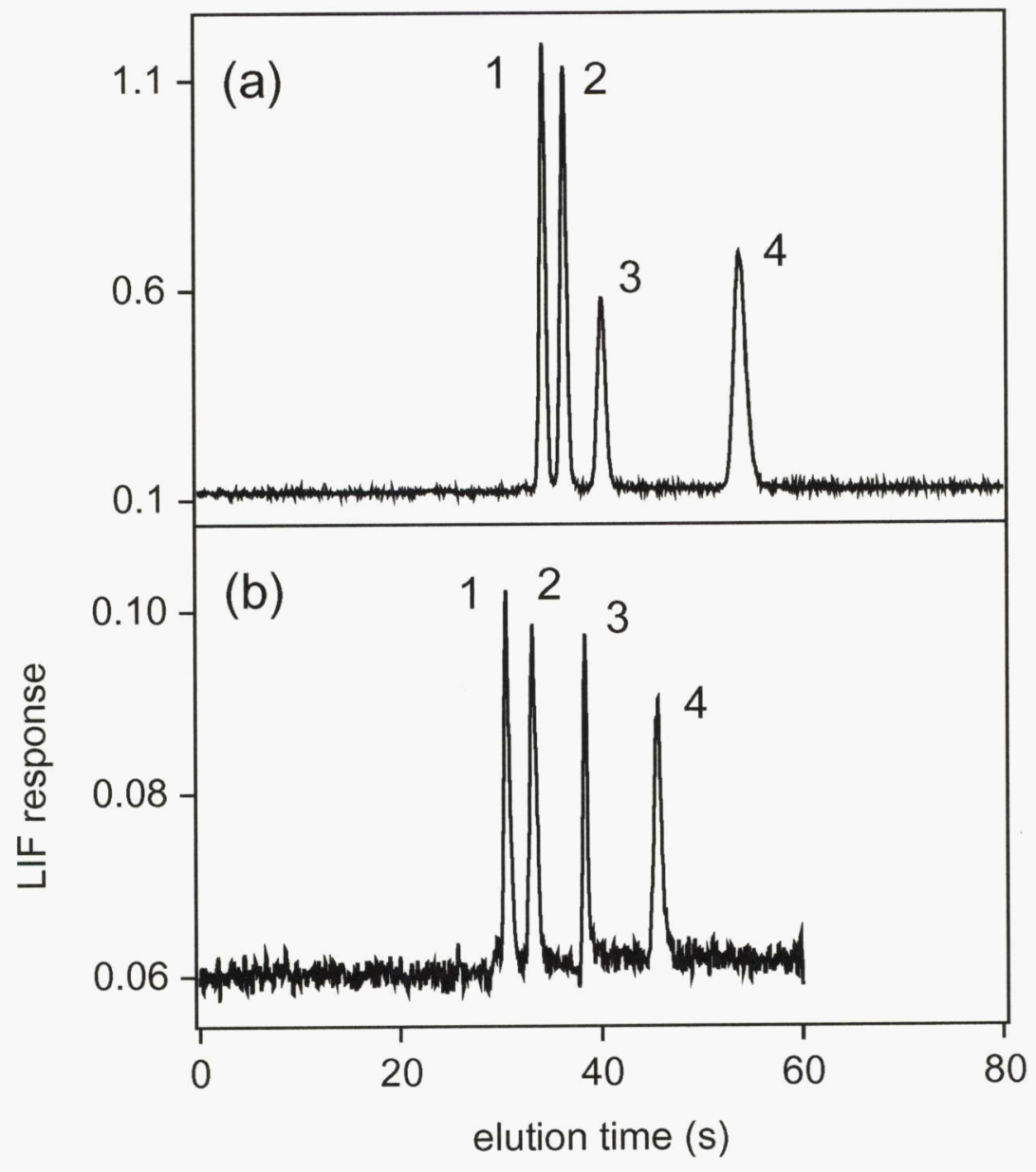

Figure 4. Separation of (1) anthracene, (2) pyrene, (3) 1,2-benzofluorene, and (4) benzo(a)pyrene. Chip 1 and a linear gradient were used, and the injection time was $20 \mathrm{~s}$. The concentrations of analytes $1,2,3$, and 4 were 2.8, 0.9, 5.8, and $5.0 \mu \mathrm{M}$ in (a), and $100,28,230$, and $200 \mathrm{nM}$ in (b), respectively. 


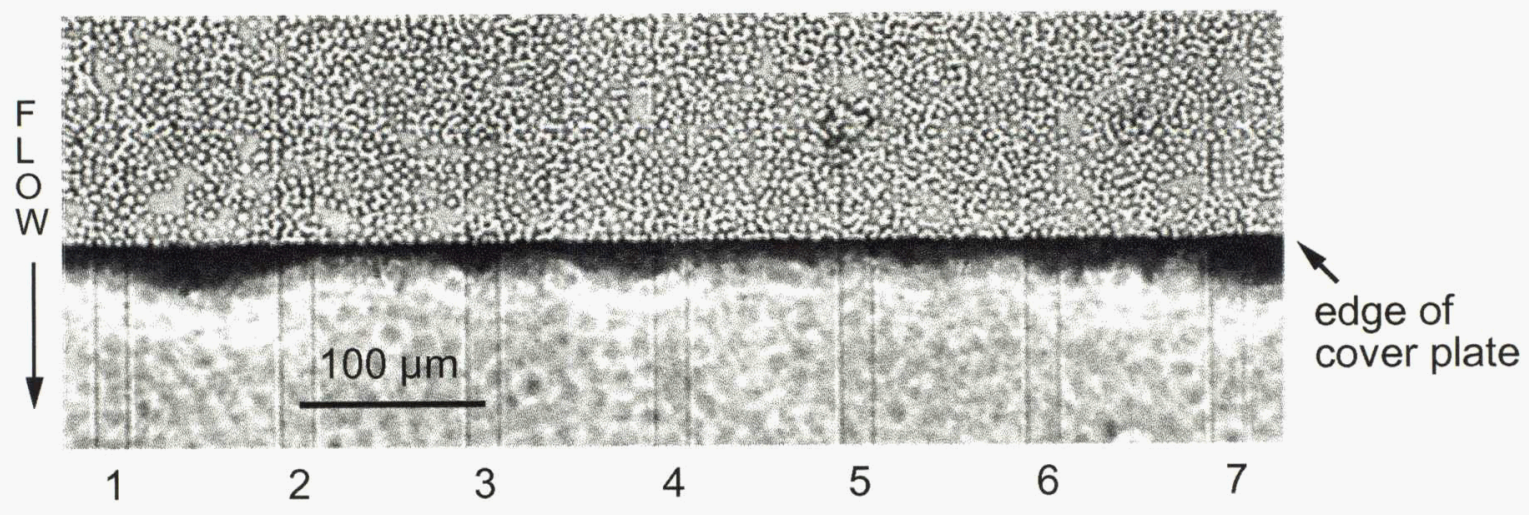

Figure 5. Composite photograph of the seven parallel channels comprising the filter element with $5 \mu \mathrm{m}$ diameter silica particles excluded. Particle collection time was 5 min. 


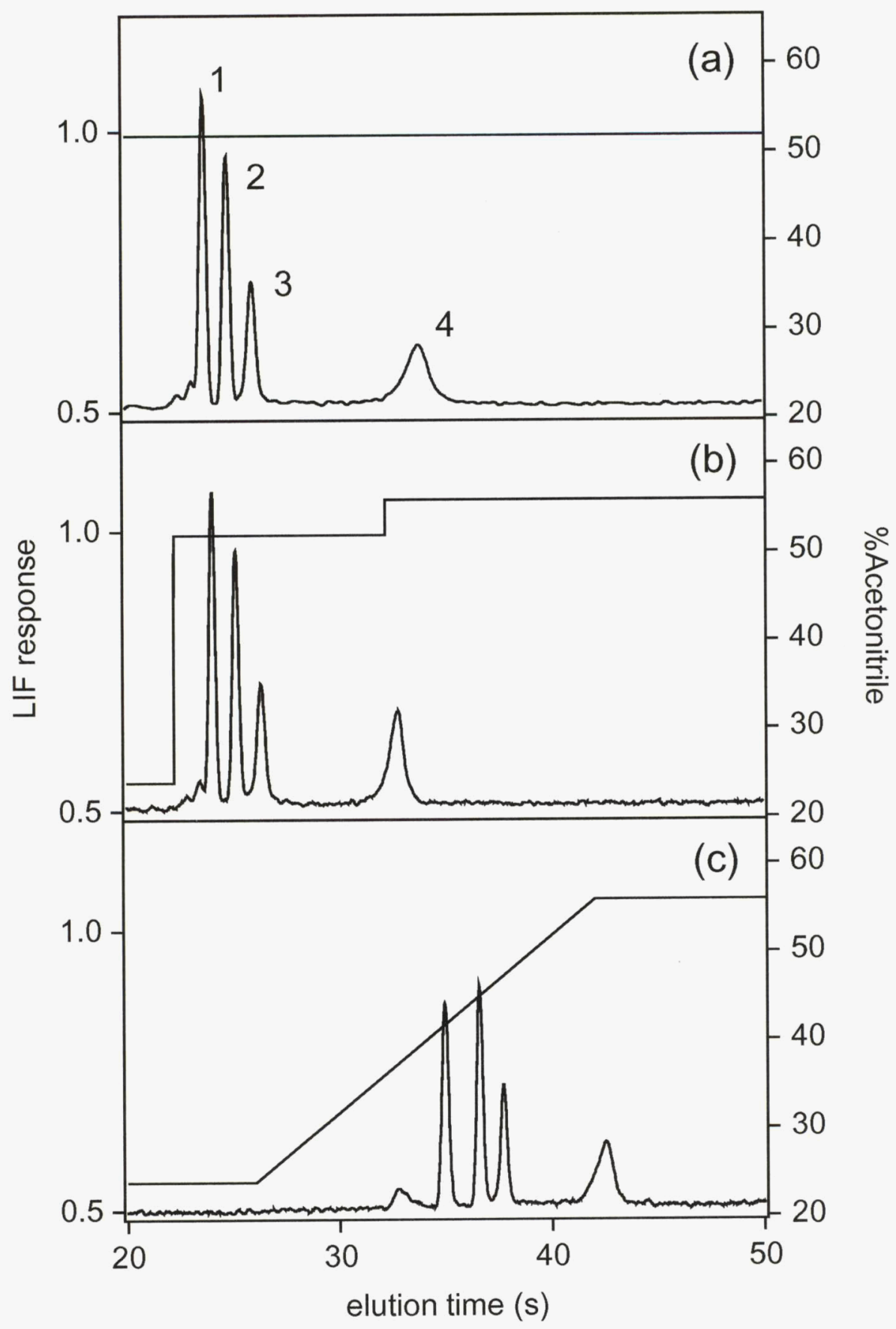

Figure 6. (a) Isocratic, (b) step gradient, and (c) linear gradient separations of (1) anthracene, (2) pyrene, (3) 1,2-benzofluorene, and (4) benzo(a)pyrene with $5 \mu \mathrm{m}$ silica particles in the sample. Chip 2 was used, and the injection time was $20 \mathrm{~s}$.

The concentrations of analytes $1,2,3$, and 4 were $2.8,0.9,5.8$, and $5.0 \mu \mathrm{M}$, respectively. The dashed lines show the gradient profiles as seen at the detector. 


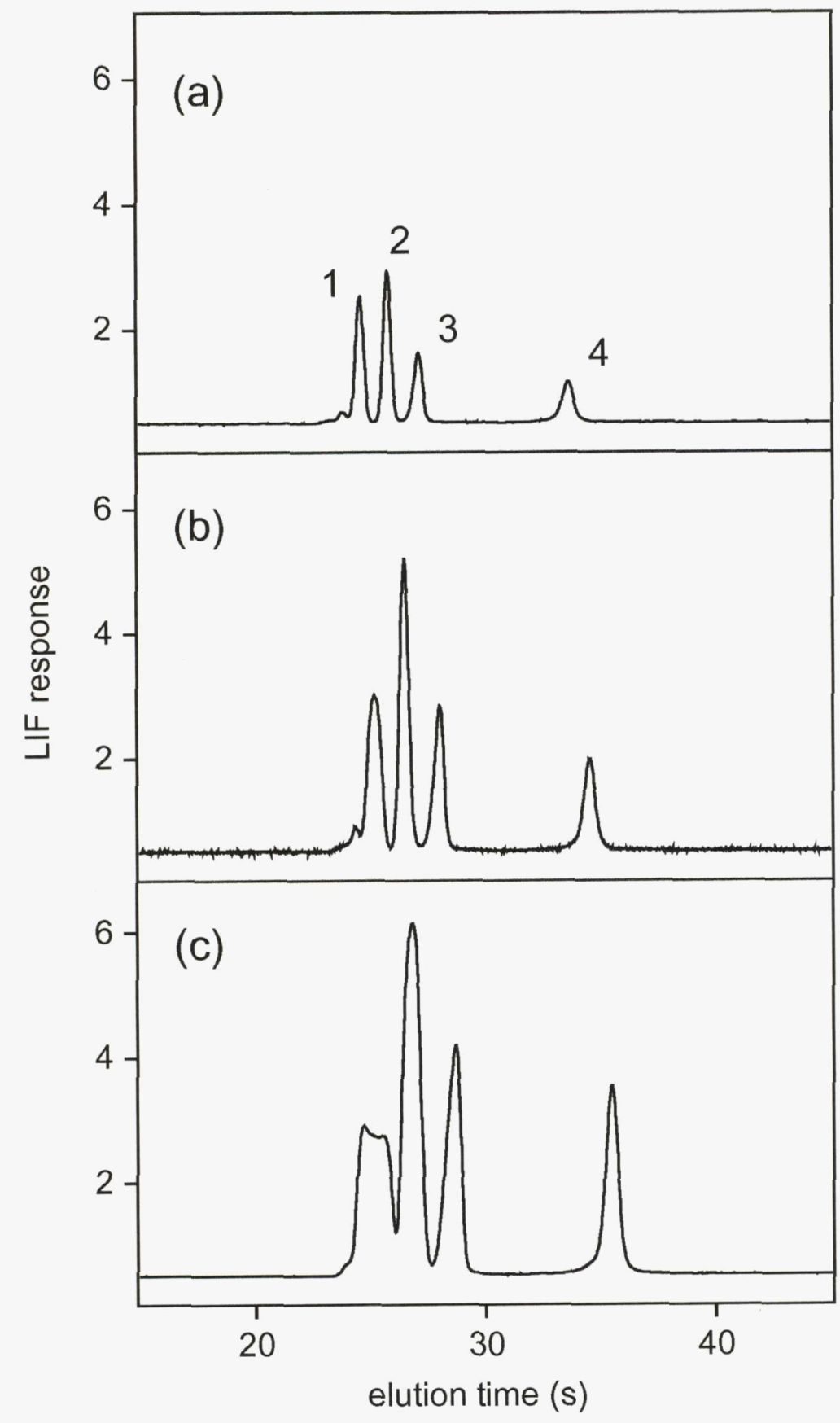

Figure 7. Separations of (1) anthracene, (2) pyrene, (3) 1,2-benzofluorene, and (4) benzo(a)pyrene for injection times of (a) 80, (b) 160, and (c) $320 \mathrm{~s}$ with $5 \mu \mathrm{m}$ silica particles in the sample. Chip 2 and a step gradient were used. The concentrations of analytes $1,2,3$, and 4 were $2.8,0.9,5.8$, and $5.0 \mu \mathrm{M}$, respectively. 


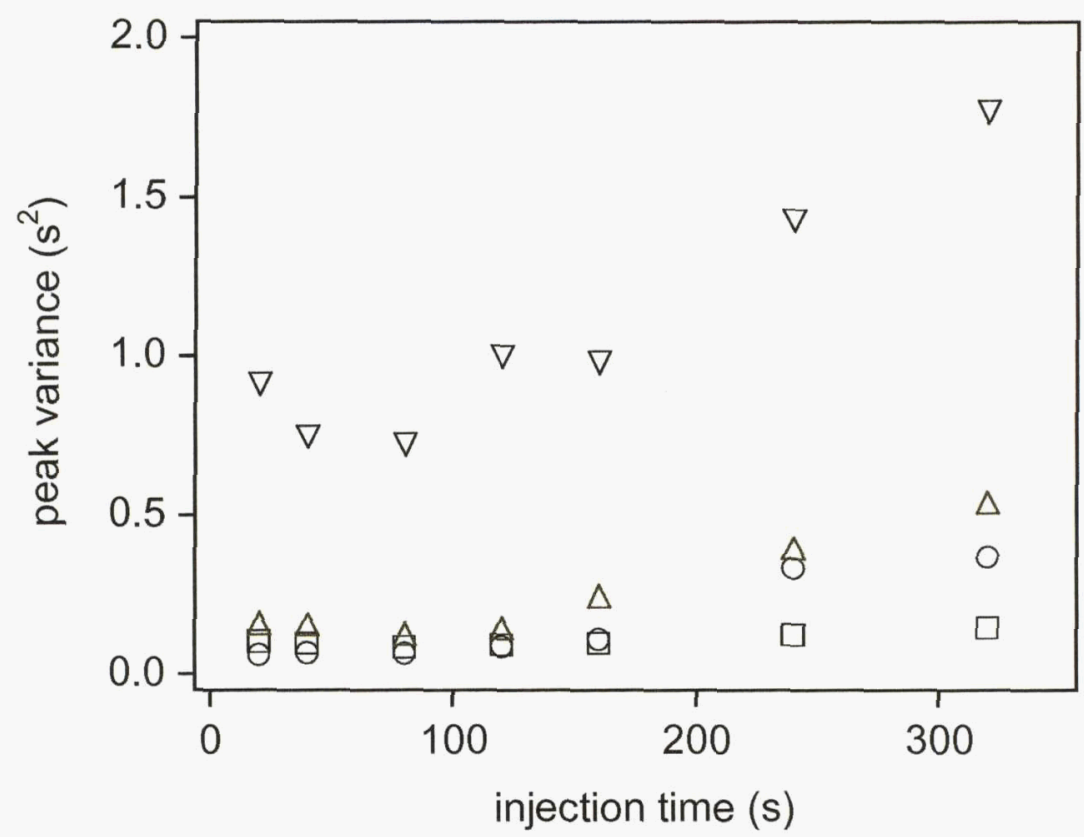

Figure 8. Peak variance with concentration time for anthracene (O), pyrene ( $\square$ ), 1,2-benzofluorene $(\Delta)$, and 1,2-benzo(a)pyrene $(\nabla)$. Data include Figure 7. 